\title{
Design and application of chitosan microspheres as oral and nasal vaccine carriers: an updated review
}

This article was published in the following Dove Press journal:

International Journal of Nanomedicine

12 December 2012

Number of times this article has been viewed

Mohammad Ariful Islam ${ }^{1-3, *}$

Jannatul Firdous ${ }^{1-3, *}$

Yun-Jaie Choi'

Cheol-Heui Yun ${ }^{1-4}$

Chong-Su Cho ${ }^{1,2}$

'Department of Agricultural

Biotechnology, ${ }^{2}$ Research Institute for Agriculture and Life Sciences, ${ }^{3}$ Center for Food and Bioconvergence, ${ }^{4}$ World Class University Biomodulation Program, Seoul National University, Seoul, South Korea

*These authors contributed equally to this work

\begin{abstract}
Chitosan, a natural biodegradable polymer, is of great interest in biomedical research due to its excellent properties including bioavailability, nontoxicity, high charge density, and mucoadhesivity, which creates immense potential for various pharmaceutical applications. It has gelling properties when it interacts with counterions such as sulfates or polyphosphates and when it crosslinks with glutaraldehyde. This characteristic facilitates its usefulness in the coating or entrapment of biochemicals, drugs, antigenic molecules as a vaccine candidate, and microorganisms. Therefore, chitosan together with the advance of nanotechnology can be effectively applied as a carrier system for vaccine delivery. In fact, chitosan microspheres have been studied as a promising carrier system for mucosal vaccination, especially via the oral and nasal route to induce enhanced immune responses. Moreover, the thiolated form of chitosan is of considerable interest due to its improved mucoadhesivity, permeability, stability, and controlled/extended release profile. This review describes the various methods used to design and synthesize chitosan microspheres and recent updates on their potential applications for oral and nasal delivery of vaccines. The potential use of thiolated chitosan microspheres as next-generation mucosal vaccine carriers is also discussed.
\end{abstract}

Keywords: chitosan microspheres, oral, nasal, vaccine delivery, mucosal and systemic immune responses

\section{Introduction}

Vaccination is cost-effective, and probably the best preventable strategy against most diseases. ${ }^{1}$ Traditionally, vaccines are administered parenterally via an intramuscular or subcutaneous route. ${ }^{2,3}$ This process of vaccine delivery incurs difficulties such as needle phobia, low patient compliance, short half-life, potential contamination while using needles, and a necessity for highly trained personnel. As a result, oral and nasal vaccination has been paid considerable attention as a way to overcome such potential drawbacks and eliminate the problems associated with parenteral administration of vaccines. ${ }^{4}$ Better yet, parenteral vaccination mostly stimulates systemic immunity, whereas mucosal vaccination tends to confer both systemic and mucosal immune responses. ${ }^{5}$ In regard to mucosal administration of protein drugs or vaccines, microspheres are well known for their controlled delivery formulation, ${ }^{6-8}$ which would provide a longlasting boosting effect and enhance the effectiveness of the immune response against infectious diseases. ${ }^{8}$

Chitosan has well-defined properties including bioavailability, biocompatibility, low cost, and an ability to open the intracellular tight junction; therefore, it has been suggested as a suitable polymeric material for mucosal delivery. ${ }^{9}$ Desirable properties 
of chitosan can be determined from its molecular weight (MW) and degree of deacetylation (DD). It has been reported that high MW chitosan enhances the absorption of various compounds across the mucosal barrier. ${ }^{9,10}$ Due to its cationic property, positively charged chitosan would have an electrostatic interaction with the negatively charged mucosal surface. ${ }^{11}$ Moreover, chitosan possesses mucoadhesivity, beneficial for prolonging the retention time at the mucosal area for a controlled and sustained therapeutic effect. ${ }^{4}$ Nontoxicity is another prerequisite property of chitosan, which can be effectively applied for mucosal delivery of vaccines as a form of the microparticulate system. In an aqueous environment, chitosan swells and forms a gellike layer, favorable for the interaction of polymers with glycoprotein in mucous. In the case of nasal delivery, chitosan possesses good bioadhesive properties and can reduce the rapid clearance of vaccine from the nasal cavity where it could be delivered to nasal-associated lymphoid tissue - the induction and effector sites for vaccine-induced immune responses. ${ }^{11}$

\section{General aspects of chitin and chitosan}

Chitin is an abundant source of chitosan, a unique cationic polysaccharide superior to any man-made cationic derivatives. ${ }^{12}$ In general, it comprises the skeletal materials in invertebrates. It is also found in egg shells of nematodes and rotifer as well as in the cuticles of arthropods, exoskeletons, peritrophic membranes, and cocoons of insects. In the fungal walls, chitin varies in crystallinity, degree of covalent bonding to other wall components, and DD. ${ }^{12}$ It was reported as the principal component of protective cuticles of crustaceans such as crabs, shrimps, prawns, and lobsters. ${ }^{11}$

Chitosan, a natural linear polyaminosaccharide obtained by alkaline deacetylation of chitin, is the second most abundant polysaccharide next to cellulose. ${ }^{12}$ It is made up of copolymers of glucosamine and $\mathrm{N}$-acetyl-glucosamine, while chitin is a straight homopolymer composed of $\beta$ - $(1,4)$-linked $\mathrm{N}$-acetyl-glucosamine units. ${ }^{13-15}$ Chitosan has one primary amino and two free hydroxyl groups for each C6 building unit (Figure 1). Due to the presence of abundant amino groups, chitosan carries a positive charge and thus reacts with negatively charged polymers as well as with mucosal surfaces, making it a useful polymer for mucosal delivery. ${ }^{11}$ Many studies have reported the use of chitosan in the formation of gels, nanoparticles, and microspheres for drug delivery application. $^{12}$

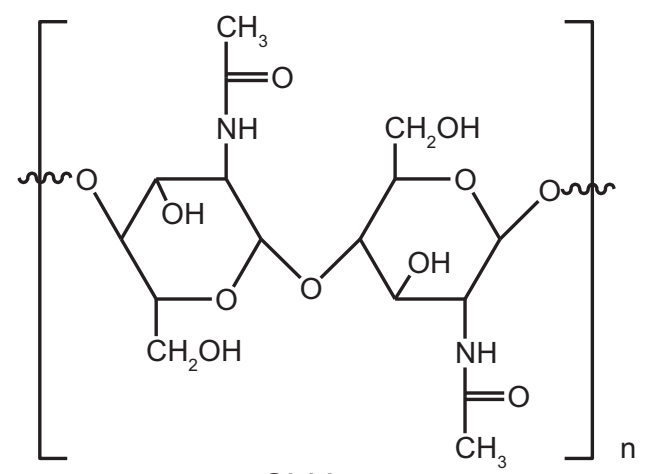

Chitin

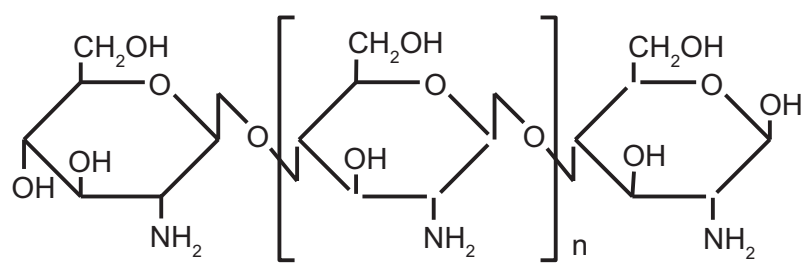

Chitosan

Figure I Structure of chitin and chitosan.

\section{Chitosan microspheres (CMs)}

Extensive research has been carried out to exploit the use of chitosan as a drug or vaccine carrier. ${ }^{11}$ Indeed, chitosan has been used for prolonged and targeted delivery of drug and macromolecules. CMs can be a better option due to their ability for sustained release and improved bioavailability of target molecules. CMs also enhance the uptake of hydrophilic substances across epithelial cells. ${ }^{11}$ It has been reported that a strong interaction between cationic CMs and anionic glycosaminoglycan receptors can retain the microspheres at the target site of the capillary region. ${ }^{16} \mathrm{CMs}$ have been applied in the oral, ${ }^{9}$ parenteral, ${ }^{17}$ and nasal delivery ${ }^{10,18,19}$ of encapsulated vaccine, DNA, or small interfering ribonucleic acid transfection studies. ${ }^{20-23}$

\section{Biodegradability, biocompatibility, and safety of CMs}

Biodegradability and biocompatibility play important roles in the metabolic process of chitosan in the body. It has been suggested that for systemic absorption a suitable MW (30-40 kDa) is essential for renal clearance dependent on the type of the polymer. ${ }^{24}$ When the size of the polymer is larger than this range, then degradation is necessary for the polymer to be eliminated from the body. Degradation of chitosan is known to occur in vertebrates by lysosomes and several bacterial enzymes. ${ }^{24}$ The biodegradability of chitosan 
in living organisms is dependent on its DD of chitin wherein the degradation rate decreases with an increase in DD. ${ }^{25,26}$ Primarily, chitosan is degraded sufficiently and eliminated properly in most cases when given adequate conditions. ${ }^{27}$ Chitosan, as any other drug delivery materials, should be preferentially degraded after the efficient delivery of vaccine to the target site. The digestion of chitosan was found to be species-dependent and also dependent on the availability of the amine group in the composition of chitosan. ${ }^{27}$

The safety of chitosan has been extensively studied and it was found that it is a biologically compatible polymer with a minimal toxicity. ${ }^{28,29}$ Many countries including Japan, Italy, and Finland have approved the use of chitosan for dietary application..$^{30}$ It has also been approved by the Food and Drug Administration for wound dressing application in the USA. ${ }^{31}$ As chitosan is considered a nontoxic and nonirritant material, it is widely applied as a potential excipient in pharmaceutical formulations as well as in cosmetic industries. It is biocompatible for both healthy and infected skin. ${ }^{32}$ It has been described that the median lethal dose for an oral administration of chitosan in rodents was $>16 \mathrm{~g} / \mathrm{kg},{ }^{31}$ suggesting that it is safe and the risk of side effects after oral administration is negligible. On the other hand, Dash et al found that the toxicity of chitosan was dependent on its DD and MW. ${ }^{24}$ As MW and concentration increased, the toxicity of chitosan also increased. It was noted that the toxicity of high DD chitosan was greatly increased by changes in MW and concentration when compared to that of low DD. Interestingly, chitosan and its derivatives were toxic to several bacteria, fungi, and parasites. ${ }^{33-35}$ This could be beneficial to controlling infectious diseases; however, the precise mechanism behind this inhibitory effect is yet to be further examined. It has been reported that no significant pyrogenic and toxic effects of chitosan were found in mice, rabbits, and guinea pigs. ${ }^{36}$ In a fat chelation study, $4.5 \mathrm{~g} /$ day chitosan in humans was reported to be nontoxic. ${ }^{37}$ It was noted, however, that in both of these studies the MW and DD were not specified. . $^{36,37}$ It has been reported that chitosan nanoparticles with $80 \mathrm{kDa}$ MW and $80 \%$ DD showed no toxicity in mice when orally delivered at $100 \mathrm{mg} / \mathrm{kg} .{ }^{38}$ Moreover, chitosan solution exposed to nasal mucosa showed no significant changes in mucosal cell morphology compared to the control..$^{10}$ Collectively, chitosan exhibits minimal toxicity and side effects, which opens the possibility for its application and adoption in vaccine delivery as a safe and biocompatible material.

\section{Bioavailability of CMs}

Most vaccines are administered by parenteral injection because the bioavailability of mucosally delivered vaccines via the oral or nasal route is generally low. ${ }^{39}$ These vaccines are sometimes impermeable to the mucosal barrier owing to their large MW and hydrophilic characteristics. Moreover, they can be easily degraded by the proteolytic enzymes present at the mucosal site. On the other hand, parenteral injections require a relatively high dose because the in vivo half-life of the vaccine is generally no more than a few hours which is considered one of the major problems of parenteral administration. ${ }^{39}$ Thus, an improved system that can provide a sustained and controlled delivery of vaccine with maximum bioavailability is a priority.

Chitosan is not only nontoxic and biodegradable but it also exhibits excellent mucoadhesive properties and permeation-enhancing effect of the delivery materials across the cell surface, especially the mucosal area. ${ }^{39} \mathrm{CMs}$ also have potential applications for enhancing the adsorption of mucosally administered biomacromolecules through the paracellular route. ${ }^{40,41}$ They have the potential to loosen up the tight junction between epithelial cells and to reduce transepithelial electrical resistance. ${ }^{42}$ It is worthwhile mentioning that mucoadhesivity is another potential benefit to using CMs for improved drug adsorption because cationic chitosan interacts with the anionic mucosal layer, which has sialic acid moieties. This adhesivity offers various advantages for an enhanced uptake of the therapeutic vaccines at the site of the induction phase: (1) mucoadhesive CMs could strongly reduce degradation of the vaccine by proteases at the absorption membrane by providing an intimate interaction with intestinal mucosa; (2) the adhesion of vaccine-loaded $\mathrm{CMs}$ to the mucosal layer provides an excessive driving force by a high concentration gradient towards the absorption membrane, leading to enhanced paracellular uptake; and (3) the mucoadhesive properties of chitosan provides a prolonged residual time of CMs on mucosal tissue, leading to drug absorption for an extended period of time and thus improving its bioavailability. ${ }^{40,41}$ Patil et al found a strong interaction between mucin in the nasal mucus layer and CMs, which resulted in rapid absorption and high bioavailability. Moreover, CMs were cleared slowly from the nasal cavity, also improving bioavailability. ${ }^{43}$ In another study, Wang et al emphasized the enhancement of drug bioavailability using both the mucoadhesivity and permeation-enhancing effect of $\mathrm{CMs},{ }^{39}$ suggesting that $\mathrm{CMs}$ could not only protect vaccines from degradation but also improve permeation, uptake, and bioavailability of the drug. They further defined the parameters, such as size and distribution, of CMs that are important for improving drug bioavailability, reproducibility, and repeatability as well as steady release behavior. ${ }^{39}$ 
Producing equal sized CMs is very difficult, and the size distribution would be too broad if the microspheres are prepared by mechanical stirring or ultrasonication technique, which are common methods for CM preparation. ${ }^{44}$ These could limit their vaccine delivery application. Firstly, the poor reproducibility of equal sized CMs may result in poor repeatability on release behavior and efficacy among the different batches. Secondly, the therapeutic efficacy can hardly be achieved with irregular sized CMs and a broad size distribution. Thirdly, a broad size distribution of CMs would result in poor bioavailability of the vaccine. Fourth, the side effects of vaccine therapy would likely be increased. ${ }^{44}$ Therefore, particle size is an important factor that should be taken into account in the application and pharmacodynamic effect of vaccine-loaded CMs. Thus, it is important to prepare CMs of uniform size with a narrow size distribution and controlled release profile for their effective application in mucosal vaccine delivery.

\section{Low off-target immunogenicity of CMs}

One of the major concerns of a vaccine carrier system is the unwanted immunogenicity and pathogenicity caused by off-target reactions between the carrier itself and the body's immune system. ${ }^{45}$ This is the major disadvantage of using bioengineered viruses or bacteria as delivery vehicles for vaccines. ${ }^{45}$ Therefore, polymeric carriers have been investigated as a useful alternative for the efficient delivery of vaccines without unwanted immunological outcomes. In this regard, chitosan can be considered a powerful polymer candidate because it has enormous potential for use as a vaccine carrier system that possesses low off-target immunogenicity, ${ }^{46}$ suggesting that it will limit unwanted off-target immune reactions with the body's normal immune function and not interfere with the actual vaccine-mediated immune response which is to be loaded. Several reports have also suggested that chitosan and its derivatives could be useful for drug delivery application without any significant offtarget immunogenicity. ${ }^{47,48}$ Therefore, CMs (without vaccine loaded) are expected to neither alter normal immunological activity and biological function in the body nor interfere with the vaccine efficacy by showing unwanted off-target immunogenicity.

\section{Preparation of CMs}

Different methods have been studied and applied to prepare CMs for the delivery of drugs and vaccines. Several methods are discussed here in detail and are summarized in Table 1.

\section{Interaction with anions}

\section{lonotropic gelation}

The counterions that are used in the ionotropic gelation method can be divided into two main categories: low MW counterions (eg, pyrophosphate, tripolyphosphate, tetrapolyphosphate, octapolyphosphate, hexametaphosphate, octyl sulfate, lauryl sulfate, hexadecyl sulfate, and cetyl stearyl sulfate) and high MW counterions (eg, alginate, $\kappa$-carrageenan, and polyaldehydrocarbonic acid). Briefly, chitosan solution is added dropwise into magnetically stirred aqueous counterions. The beads are removed from the solution by filtration, washed with distilled water, and dried. ${ }^{11}$ CMs encapsulated with an atrophic rhinitis vaccine prepared by ionotropic gelation were nasally administered, which enhanced cytokine (tumor necrosis factor- $\alpha$ [TNF- $\alpha]$ ) and nitric oxide production as an indication of immune stimulating activity. ${ }^{49}$

\section{Emulsification and ionotropic gelation}

In the emulsification and ionotropic gelation method, an aqueous solution of chitosan is added to a nonaqueous continuous phase (isooctane and emulsifier) to form a waterin-oil emulsion. Sodium hydroxide solution is then added at different intervals, leading to ionotropic gelation. The microspheres, thus formed, are removed by filtration, washed, and then dried..$^{50}$ It has been suggested that the conventional emulsification and ionotropic gelation method for preparation of CMs provides irregular microparticles, whereas spherical microparticles with a diameter of about $10 \mu \mathrm{m}$ can be obtained when employing a modified process. In one modified process, gelatin is used, which allows the ionic crosslinking of chitosan/gelatin (water-in-oil emulsion) to take place under coagulation conditions at a low temperature. ${ }^{51}$ Several other crosslinking agents have been used for surface modification of chitosan/gelatin microspheres: the surface was very smooth in sodium sulfate or sodium citrate crosslinked chitosan/gelatin microspheres; however, large gaps were observed in chitosan/tripolyphosphate microspheres. ${ }^{51}$ It has been reported that the increase of stirring speed leads to a decrease in diameter and a narrower size distribution. ${ }^{51}$

\section{Complex coacervation}

Sodium alginate, sodium carboxymethyl cellulose, $\kappa$-carrageenan, and sodium polyacrylic acid can be used for complex coacervation with chitosan to form microspheres after the interionic interaction between oppositely charged polymers. For example, potassium chloride and calcium chloride were used to formulate the coacervate capsules of 


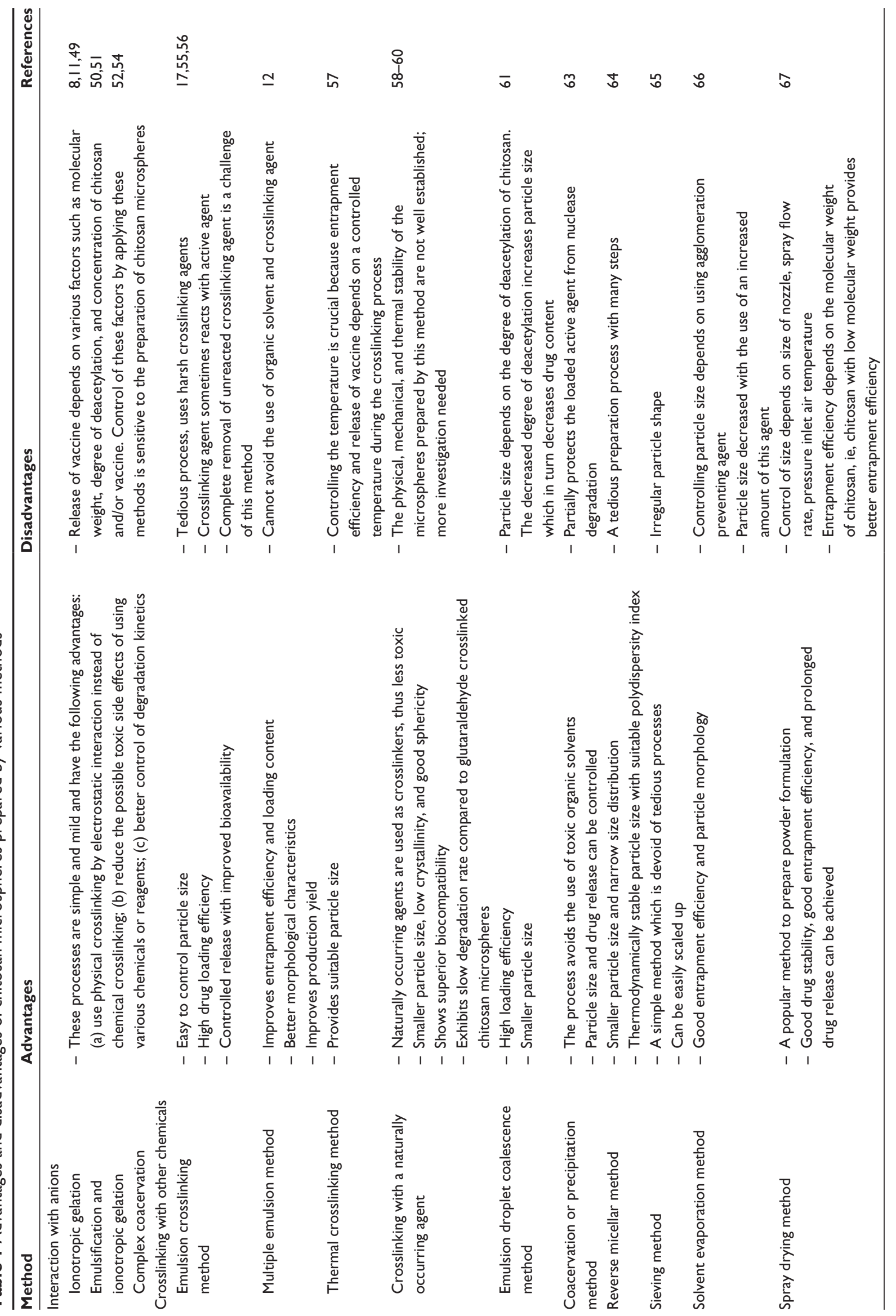


chitosan-alginate and chitosan- $\kappa$-carrageenan, respectively, and the obtained capsules were hardened in the counterion solution before washing and drying. ${ }^{52-54}$

\section{Crosslinking methods}

\section{Emulsion crosslinking method}

Water insoluble reagents can be simply dispersed in chitosan solution and entrapped by the emulsion crosslinking process. Glutaraldehyde, formaldehyde, and genipin have been widely used as crosslinking agents for the preparation of CMs. In the emulsion crosslinking method, chitosan solution is first prepared by dissolving chitosan with acetic acid. This solution is then added to liquid paraffin containing a surfactant, forming a water-in-oil emulsion before the addition of a crosslinking agent. The formed microspheres are filtered, washed with suitable solvent, and dried. ${ }^{17,55,56}$

\section{Multiple emulsion method}

The multiple emulsion method is probably the best way to increase the entrapment efficiency of the target molecule in CMs. In this method, a primary emulsion (oil-in-water) is first formed (nonaqueous solution containing the target molecule in chitosan solution). This primary emulsion is then added to an external oil phase to form multiple emulsions (oil-in-water-in-oil) followed by either the addition of glutaraldehyde (as a crosslinking agent) or the evaporation of an organic solvent. ${ }^{12} \mathrm{CMs}$, loaded with hydrophobic reagents, were found to have better morphological characteristics and yield when prepared by the multiple emulsion method. ${ }^{12}$

\section{Thermal crosslinking method}

In the thermal crosslinking technique, CMs are prepared with different thermal conditions in various steps. Orienti et al reported $\mathrm{CM}$ preparation by the thermal crosslinking method using citric acid, which served as crosslinking agent. ${ }^{57} \mathrm{Citric}$ acid was added to chitosan solution in acetic acid $(2.5 \%$ weight/volume) and then cooled to $0^{\circ} \mathrm{C}$ before adding to corn oil. After stirring for 2 minutes, the emulsion was then added dropwise to corn oil by maintaining the temperature at $120^{\circ} \mathrm{C}$. Then, the crosslinking was performed under vigorous stirring (1000 rpm) for 40 minutes and the microspheres obtained were filtered, washed, dried, and sieved. ${ }^{57}$

\section{Crosslinking with a naturally occurring agent}

Genipin, a naturally occurring crosslinking agent, has also been used to prepare CMs by the spray drying method, which provides small particle size, low crystallinity, and good sphericity. ${ }^{58}$ It was reported that genipin crosslinked
CMs had better biocompatibility and slower degradation rate than glutaraldehyde crosslinked CMs. ${ }^{59,60}$ The microspheres used as an injectable chitosan-based drug delivery system revealed low toxicity.

\section{Emulsion droplet coalescence method}

Tokumitsu et al developed the emulsion droplet coalescence method for CM preparation, which implements the principle of both emulsion crosslinking and precipitation. ${ }^{61}$ In this method, precipitation is usually induced by coalescence of chitosan droplets with sodium hydroxide. Briefly, a drug containing stable emulsion solution of chitosan is prepared in liquid paraffin oil. This emulsion is mixed with another stable emulsion containing a chitosan aqueous solution of sodium hydroxide with high-speed stirring, which allows the droplets of each emulsion to collide randomly and coalescently. This results in the precipitation of chitosan droplets with small particle size. CMs loaded with gadopentetic acid were prepared using this method for gadolinium neutron capture therapy. ${ }^{61}$ Gadopentetic acid interacts electrostatically with amino groups of chitosan since it is a bivalent anionic compound. A range of nanosized particles and a high loading of gadopentetic acid were obtained through this emulsion droplet coalescence method compared to the conventional emulsion crosslinking method. ${ }^{61}$

\section{Precipitation or coacervation method}

Chitosan precipitates when it interacts with an alkaline solution since it is not soluble in an alkaline $\mathrm{pH}$ medium. In this method, chitosan particles are prepared by dropping chitosan solution into an alkaline solution (eg, sodium hydroxide, sodium hydroxide-ethanediamine, or sodium hydroxide-methanol) through a compressed air nozzle, which produces coacervate droplets. Particles are collected by precipitation or centrifugation before excessive washing with hot and cold water, respectively. ${ }^{62}$ The particle sizes can be controlled by varying the diameter of the compressed air nozzle together with the pressure. A crosslinking agent can also be used to harden the particles, ${ }^{62}$ which would be beneficial because of its slow release. Sodium sulfate was also used to prepare CMs using this precipitation technique. Recombinant human interleukin-2-loaded CMs were prepared by a dropwise addition of sodium sulfate-containing recombinant human interleukin-2 solution in acidic chitosan solution. As a result, chitosan was precipitated and recombinant human interleukin-2 was incorporated when CMs were formed. ${ }^{63}$ Of note, this method is devoid of any crosslinking agent. 


\section{Reversed micellar method}

Reverse micellar is the stable liquid mixture of oil, water, and surfactants dissolved in organic solvents. To this mixture, an aqueous solution of chitosan and the target molecule are added before the addition of a crosslinking agent such as glutaraldehyde. ${ }^{62}$ Mitra et al described the preparation of doxorubicin-dextran conjugate-encapsulated chitosan nanoparticles. ${ }^{64}$

\section{Sieving method}

Agnihotri and Aminabhavi developed a method to prepare clozapine-loaded CMs. ${ }^{65}$ In this method, a thick jelly mass of chitosan was prepared in $4 \%$ acetic acid and crosslinked with glutaraldehyde. The crosslinked nonsticky jelly mass was passed through a sieve to get microparticles of a suitable size, which were then washed with $0.1 \mathrm{~N}$ sodium hydroxide to remove unreacted glutaraldehyde and dried overnight at $40^{\circ} \mathrm{C}$. As a result, a high loading efficiency of clozapine $(98.9 \%)$ was achieved. However, the particles were irregular in shape with an average size of 543-698 $\mu \mathrm{m}$. The irregular shape and size of the particles is one of the major disadvantages of this method, which could affect the bioavailability of $\mathrm{CMs}$ in vivo. However, an in vitro and in vivo study demonstrated a controlled and sustained release of the drug. ${ }^{65}$

\section{Solvent evaporation method}

The solvent evaporation method involves the formation of emulsion between a polymer solution and an immiscible continuous phase - either aqueous (oil-in-water) or nonaqueous (water-in-oil). This can be done by using liquid paraffin/acetone. The target molecule dissolved in acetone is dispersed in chitosan solution and the mixture is emulsified in liquid paraffin while stirring. The microsphere suspension is filtered, washed, and dried. Magnesium stearate can be added as an agglomeration preventing agent. It appears that the average particle size decreases when the amount of magnesium stearate used in the preparation is increased. ${ }^{66}$

\section{Spray drying method}

Spray drying is one of the most widely investigated methods of preparing CMs in which chitosan solution is sprayed and then air-dried followed by the addition of a crosslinking agent. He et al prepared CMs by spray drying multiple emulsions (oil-in-water-in-oil or water-in-oil-in-water) to entrap cimetidine and famotidine into microspheres. The drug was released in a sustained and controlled fashion compared to the other microspheres prepared by traditional spray drying or the oil-in-water emulsion method. ${ }^{67}$

\section{Vaccine delivery through CMs}

CMs have been examined for the mucosal delivery of vaccines. A variety of chitosan-based carrier systems with their functional properties for oral and nasal delivery is shown in Table 2. Here, the utility of CMs for oral and nasal vaccination in vitro and in vivo are discussed.

\section{Oral delivery}

Oral delivery of vaccines has numerous advantages over conventional needle injection and is a well accepted route of vaccination. However, most vaccines are still administered by injection due to the lack of a proper delivery system to reach the induction site and to enhance the effector responses. Although oral delivery is probably the preferred administrative route of vaccines, especially for children, it causes degradation of the antigens in the gastrointestinal track and also shows inefficient targeting to the site of action when delivered in a naked form. ${ }^{68}$ Therefore, developing an effective delivery system has been considered the primary task in the oral vaccination field. To gain adequate immune responses after oral delivery, the vaccine should reach the M-cells of Peyer's patches in the gut avoiding the acidic $\mathrm{pH}$ condition of the stomach and enzymatic degradation. Even if the vaccine nearly reaches Peyer's patches, the immune response is not always induced due to the inability of antigens to gain access to Peyer's patches and because of inefficient uptake at the induction site. Several studies have shown that the uptake by M-cells was significantly enhanced and degradation of protein and peptide vaccines in the gastrointestinal track was prevented after the incorporation of vaccine with CMs. ${ }^{59,68-71}$ Due to its nontoxicity and potent antigen binding properties, chitosan has been considered a promising tool for oral vaccination. ${ }^{68}$

Extensive research on CMs for mucosal vaccine delivery, in particular, the uptake of CMs in murine Peyer's patches in vitro and in vivo, was carried out by van der Lubben et al. ${ }^{59,68,70,71}$ They prepared a human intestinal M-cell model by coculturing Caco-2 and Raji-cells and investigated the uptake of CMs.$^{59}$ No morphological changes in the monolayer were observed and this model was used to examine the in vitro uptake of CMs for oral vaccine delivery. ${ }^{59}$ They found that CMs can be taken up by the epithelium of Peyer's patches. It has been reported that the size of microparticles should be $<10 \mu \mathrm{m}$ for efficient uptake by M-cells and to reach the dome of Peyer's patches. ${ }^{3}$ Indeed, CMs used in the study were much smaller than $10 \mu \mathrm{m}$ and therefore suitable for M-cell uptake. ${ }^{68}$ Since chitosan is biodegradable, van der Lubben et al further claimed that antigen was freed from CMs after uptake by M-cells. ${ }^{68}$ 


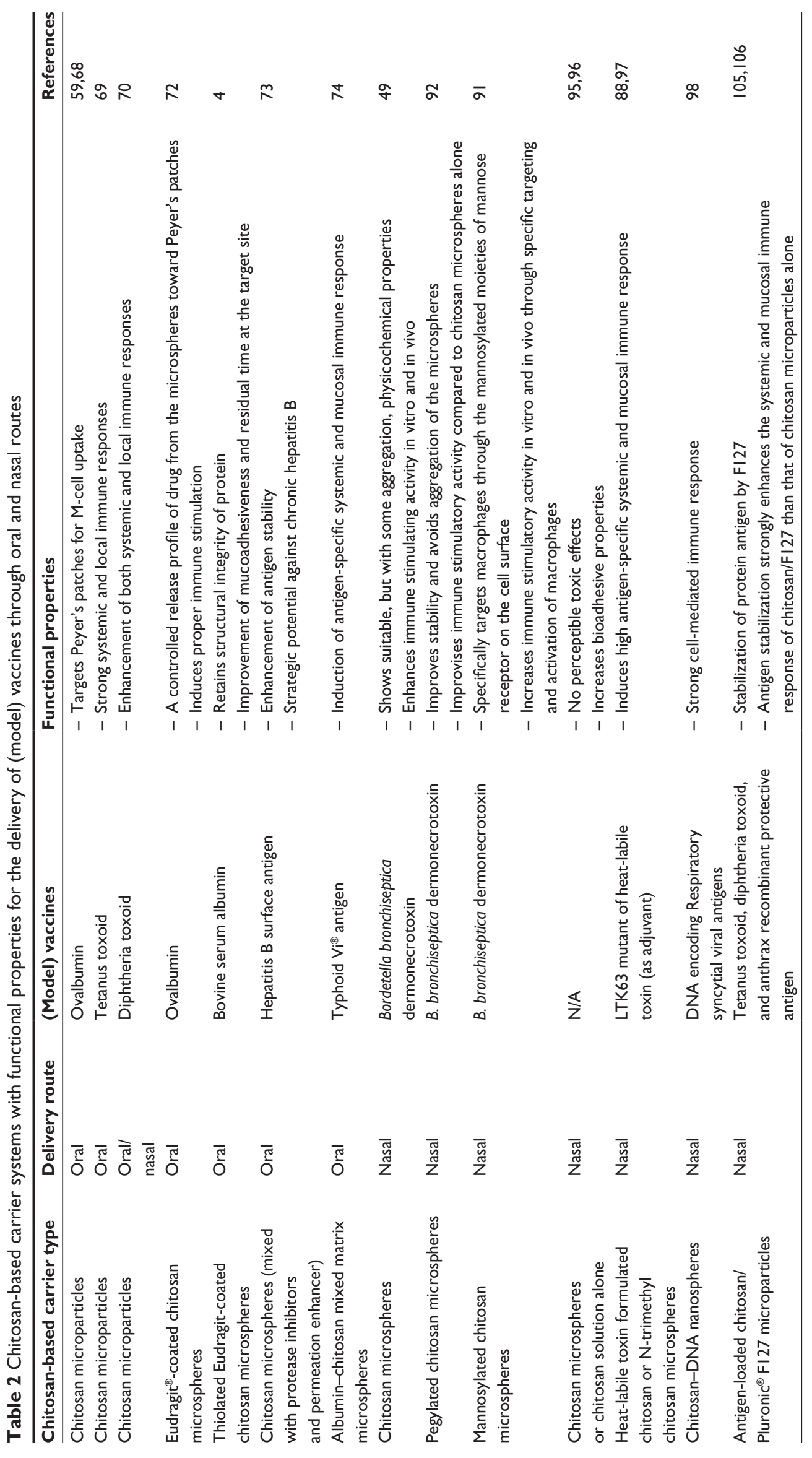


Therapeutic use of CMs for oral and nasal delivery has been examined. A diphtheria toxoid (DT) was used to examine the enhancement of both systemic and local immune responses. ${ }^{70}$ Unloaded CMs, DT-loaded CMs, and DT in phosphate-buffered saline (PBS) were delivered into mice by oral and nasal administration. DT associated with alum was subcutaneously immunized in mice as a positive control. A strong systemic and local immune response was found against DT in mice administered orally with different doses of DT-loaded CMs when compared to the mice fed with DT in PBS. Furthermore, a dose-dependent anti-DT immunoglobulin $\mathrm{G}(\mathrm{IgG})$ response in sera was found after oral administration of DT-loaded CMs. On the other hand, the systemic immune response (IgG) induced by DT-associated CMs were ten times higher than that induced with DT in PBS after nasal delivery. ${ }^{70}$

CMs were also examined after oral delivery of tetanus toxoid (TT) to induce systemic and local immune responses. ${ }^{69}$ TT-loaded CMs were prepared by the ionic crosslinking method using sodium tripolyphosphate. Unloaded CMs, TT-loaded CMs, and naked TT in PBS were orally administered in mice, and TT absorbed on aluminum phosphate was administered intramuscularly as a positive control. TT-loaded CMs enhanced a strong systemic and local immune response in a dose-dependent manner at 3 weeks after the oral delivery of vaccine compared to TT in PBS. They observed that a four-fold higher dose was needed for TT-loaded CMs to get a similar IgG response to the positive control. The study was also carried out at different time points to understand the kinetics of the immune response based on the level of IgG. It was found that the $\mathrm{IgG}$ response could be observed at day 14 and was increased after boosting at day 22 . At day 29 , the $\operatorname{IgG}$ level was lower than at day 22; however, it still maintained a higher concentration than TT in PBS at all the time points investigated. On the other hand, IgA levels were not significantly different at day four; however, the levels were significantly $(P<0.01)$ higher in TT-loaded CMs than in TT in PBS at days eight, 14 , and $22 .{ }^{69}$ These results suggest that the encapsulated vaccine in CMs enhanced the systemic as well as local immune responses compared to the nonencapsulated vaccine, rendering a safe and effective form of oral vaccination. Further studies on cellular immune responses including memory effect of B-cells and T-cells will ensure the solid effectiveness of CMs for vaccine delivery.

At first glance, chitosan would not be considered suitable for oral vaccination since it is a $\mathrm{pH}$-sensitive polymer. It is soluble at acidic $\mathrm{pH}$ and becomes insoluble at about $\mathrm{pH}$ 6.5. It has been suggested that an enteric coating can protect chitosan from the acidic stomach. ${ }^{12}$ When this reaches the intestine, the enteric layer dissolves at high $\mathrm{pH}$ and the antigen-encapsulated chitosan core is exposed to enzymes. In this state, chitosan can protect the encapsulated antigen from enzymatic degradation and most importantly can lead the antigen to reach the induction site of Peyer's patches for immune stimulation. For this, Hori et al developed Eudragit ${ }^{\mathbb{B}}$ coated CMs and evaluated ovalbumin as an oral immune delivery system. ${ }^{72}$ The ovalbumin-loaded CMs prepared by the emulsification-solvent evaporation method showed high ovalbumin content and an appropriate size for the efficient uptake by Peyer's patches. A comparable systemic $\mathrm{IgG}$ response was found after the oral administration of ovalbumin-loaded CMs in mice. Moreover, a higher intestinal mucosal IgA response was achieved using ovalbumin-loaded CMs by delivery of the microspheres toward Peyer's patches, where they were subsequently uptaken by the M-cells and the entrapped ovalbumin was released in a controlled fashion. ${ }^{72}$ In another study, Cho et al reported a mucoadhesive and $\mathrm{pH}-$ sensitive thiolated Eudragit-coated CM, designed to enhance mucoadhesivity and bioavailability of the carrier at the target site. They found strong mucoadhesive properties in vitro and in vivo, ${ }^{4}$ suggesting that Eudragit-coated CMs were a potential carrier for the oral delivery of vaccines.

Recently, hepatitis B surface antigen-loaded CMs were formulated, characterized, and optimized in vitro and in vivo for effective oral delivery of hepatitis B surface antigen against chronic hepatitis B. ${ }^{73}$ An emulsion solvent evaporation technique was applied to prepare $\mathrm{CMs}$, with the addition of protease inhibitors and permeation enhancers to overcome the limitation of the enzymatic and permeation barrier. In vitro drug release, in vivo efficacy, and importantly the effect of different storage conditions were studied to test the practicality of the system. An enhanced stability of the antigen was found when using the microspheres for a period of 4 months at room temperature, suggesting a possible way to overcome the tedious and expensive requirement of cold chain storage in the vaccine industry. Importantly, the study signifies a potential strategy for effective oral administration of hepatitis B surface antigen using the biodegradable CM system. ${ }^{73}$

Recently, Uddin et al developed an albumin-chitosan mixed matrix microsphere (ACM)-filled capsule formulation for oral administration of Typhoid $\mathrm{Vi}^{\circledR}$ antigen (TVA) to demonstrate antigen-specific systemic and mucosal immune responses..$^{74}$ TVA-loaded ACMs were filled into hard gelatin capsules with enteric coating. The physicochemical characterization such as particle size, zeta potential, swelling, 
and disintegration rates of the microspheres were favorable for oral delivery of the microencapsulated vaccine. In vivo studies showed that the oral delivery of TVA-loaded ACMs had similar IgG and IgA responses with those of the parenteral vaccination group, suggesting that TVA-loaded ACMs had the potential to induce antigen-specific immune responses when delivered via oral administration. ${ }^{74}$

\section{Nasal delivery}

Nasal administration of vaccines has been reported to enhance bioavailability and improve efficacy. ${ }^{12}$ An effective humoral and cell-mediated immune response can be achieved through nasal delivery of vaccines when the appropriate delivery system is used as a carrier for particulate antigens. ${ }^{75,76}$ Nasal-associated lymphoid tissue, present at the nasal epithelium and containing immunocompetent cells, would be an ideal target site for the nasal delivery of vaccines to induce an immune response. ${ }^{75,76}$ It has been suggested that nasal-associated lymphoid tissue epithelium has similar types of immune cells that are present in the M-cells of Peyer's patches in gut-associated lymphoid tissue and is located just below the epithelial surface, which contains macrophages, dendritic cells, lymphoid follicles (mostly B-cells), and intrafollicular areas (mostly T-cells) in a network. At these sites, particulate antigens are mainly taken up and/or transported across the cells by transcytosis without any extensive degradation. It has been well described that increased epithelial permeability influences the particulate antigen uptake across the epithelial mucosa. ${ }^{77-84}$ Importantly, chitosan has the ability to increase membrane permeability when used as a delivery system for nasal vaccination. ${ }^{85}$

However, antigen delivery through nasal administration sometimes results in poor immune responses. Several factors including limited diffusion of particulate antigens across the mucosal barrier, rapid clearance of particulate drug or vaccine formulation from the mucosal surface, and enzymatic degradation because of instability of the particulate carrier are associated with this. ${ }^{86,87}$ In order to overcome these problems, chitosan might be one of the best options for nasal administration of vaccines due to its ability to increase the retention time when it binds to the mucosal membrane. ${ }^{12}$ Several reports also demonstrated that chitosan enhanced mucosal absorption of vaccines with adjuvant activity to improve mucosal immunity after nasal administration. ${ }^{10,88,89}$

Cho and colleagues conducted extensive research on $\mathrm{CMs}$ for intranasal delivery of vaccines to induce the immune response in vitro and in vivo. ${ }^{49,90-94}$ They used Bordetella bronchiseptica dermonecrotoxin (BBD), a causative agent and a major virulence factor for atrophic rhinitis - a disease that causes huge economic damage in the swine industry. BBD-loaded CMs were prepared by the ionic gelation process using tripolyphosphate. ${ }^{49}$ The morphology of vaccine-loaded microspheres was observed as aggregated shapes, whereas unloaded microspheres were quite spherical. The average particle size of BBD-loaded CMs was $4.39 \mu \mathrm{m}$, which provides a condition for effective delivery of the vaccine to nasal-associated lymphoid tissue for immune induction. The size of unloaded CMs was about $1.94 \mu \mathrm{m}$, indicating that CMs became enlarged after vaccine loading. The release studies further demonstrated that when the MW of chitosan decreased, more BBD was released. It was also found that encapsulated BBD had greater release at higher $\mathrm{pH}$ than lower $\mathrm{pH}$. The secretion of TNF- $\alpha$ and nitric oxide from the murine macrophages treated with BBD-loaded CMs indicated that the cells stimulated with BBD-loaded CMs produced TNF- $\alpha$ and nitric oxide in a time-dependent manner at a similar level to cells stimulated with BBD alone or lipopolysaccharide. It is important to mention that BBD-loaded CMs induced a steadily increasing immune stimulating effect in the macrophages, whereas it began to decrease at 80 hours poststimulation with lipopolysaccharide. ${ }^{49}$

An in vivo study was carried out in mice that measured IgG and IgA in sera, nasal wash, and saliva after intranasal administration of BBD-loaded CMs. ${ }^{90}$ The IgA levels in nasal wash increased in a time- and dose-dependent manner after intranasal administration of BBD-loaded CMs. However, such immune response was not detected in saliva, suggesting that CMs successfully delivered the vaccine to nasal-associated lymphoid tissue after intranasal administration and induced a higher systemic and local immune response. Although in vitro and in vivo results showed $\mathrm{CMs}$ as a potential carrier for nasal delivery, BBD-loaded CMs were found in aggregated shapes because of physical and storage instabilities. ${ }^{90}$

To overcome this instability problem, chitosan was modified by covalent conjugation with polyethylene glycol to form pegylated chitosan. ${ }^{92}$ The pegylated CMs (PCMs) were prepared through a similar ionic gelation process. The average particle size of BBD-loaded PCMs was $<10 \mu \mathrm{m}$, their shape was spherical, and they were physically more stable compared to BBD-loaded CMs. Due to better stability, the vaccine was released from BBD-loaded PCMs in a more steady fashion than in BBD-loaded CMs. The study further showed that macrophages secreted TNF- $\alpha$ and nitric oxide in a time-dependent manner after exposure to BBD, BBD-loaded CMs, BBD-loaded PCMs, and lipopolysaccharide. However, a significantly higher TNF- $\alpha$ 
secretion was found in the cells treated with BBD-loaded PCMs than cells exposed to BBD-loaded CMs and BBD alone. Moreover, TNF- $\alpha$ secretion increased in a sustained fashion in the cells exposed to BBD-loaded PCMs, whereas it began to decline at 48 hours poststimulation with lipopolysaccharide. ${ }^{92}$

To increase the target specificity, another study was carried out with mannosylated CMs (MCMs) with encapsulated BBD to target macrophage mannose receptors and increase immune stimulating activity. ${ }^{91}$ Colocalization of BBD-loaded MCMs and the macrophage receptors was confirmed by confocal laser scanning microscope. The results showed that macrophages exposed to BBD-loaded MCMs secreted higher TNF- $\alpha$ and interleukin- 6 than that of BBDloaded CMs and BBD alone. Furthermore, BBD-specific $\operatorname{IgA}$ response was found to be significantly higher in saliva and serum after intranasal immunization with BBD-loaded $\mathrm{MCMs}$ in mice compared with BBD-loaded CMs, ${ }^{91}$ suggesting that the MCMs extensively assisted in stimulating macrophages for induction and enhancement of immune activity. The representative scanning electron microscope photographs of CMs and MCMs (BBD loaded and unloaded) are shown in Figure 2. ${ }^{91}$

Soane et al performed extensive research using different types of chitosan and concluded that chitosan could be

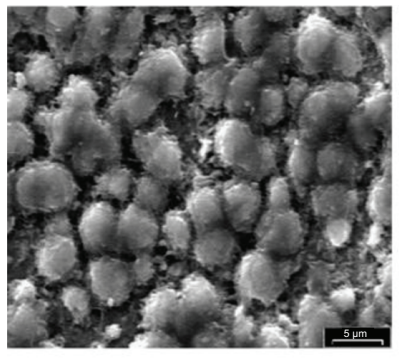

CMs

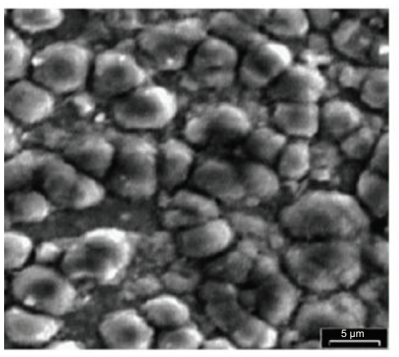

MCMs

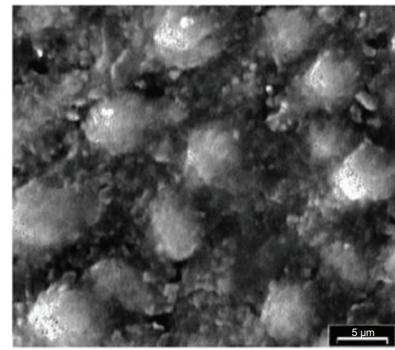

BBD-CMs

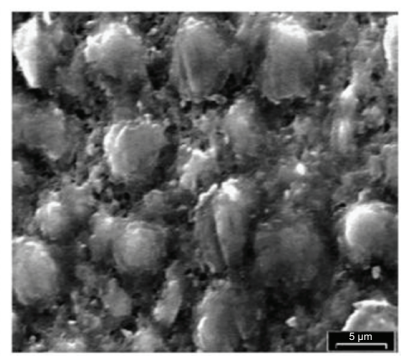

BBD-MCMs
Figure 2 Scanning electron microscope photographs of CMs, BBD-loaded CMs, MCMs, and BBD-loaded MCMs (5000x).

Notes: Bar represents $5 \mu \mathrm{m}$. Reprinted from Biomaterials, 29(12). Jiang HL, Kang ML, Quan JS, et al. The potential of mannosylated chitosan microspheres to target macrophage mannose receptors in an adjuvant-delivery system for intranasal immunization, 1931-1939. Copyright 2008 with permission from Elsevier. ${ }^{91}$

Abbreviations: BBD, Bordetella bronchiseptica dermonecrotoxin; CM, chitosan microsphere; MCM, mannosylated chitosan microsphere. used as a nasal vaccine delivery carrier without any harmful effects. ${ }^{95,96}$ To investigate this, the cilia beat frequency was studied in guinea pigs after nasal administration of chitosan solution for 28 days and found that none of the chitosan induced the changes of cilia beat frequency, indicating a safety profile of chitosan for nasal delivery. ${ }^{95}$ They further investigated the bioadhesive properties of CMs via nasal administration using three different formulations: chitosan solution, CMs, and starch microspheres, which was followed by the examination of clearance properties in human subjects. The clearance rate was 21 minutes for the control, 41 minutes for the chitosan solution, 68 minutes for the starch microspheres, and 84 minutes for the CMs. This result indicates that $\mathrm{CMs}$ have better bioadhesive properties and are able to significantly reduce the drug clearance rate and prolong the residence time of the delivered vaccine in nasal mucosa, resulting in enhanced bioavailability and efficacy. ${ }^{96}$

Several reports demonstrated the concomitant use of CMs as a mucosal adjuvant and as a vaccine delivery system. A vaccine formulation with $\mathrm{CMs}$ and a nontoxic LTK63 mutant of heat-labile toxin induced significantly higher IgG titers in sera and IgA in nasal washes after intranasal delivery in mice. ${ }^{88} \mathrm{~A}$ modified $\mathrm{N}$-trimethyl chitosan microparticulate system also showed higher antigen-specific antibody responses in sera, nasal, and vaginal wash. ${ }^{97}$ Chitosan-DNA nanospheres with intranasal delivery exhibited significant responses of cytotoxic T-cell response and interferon- $\gamma$ as well as antigen specific-IgG and $\operatorname{IgA}$, rendering a strong humoral and cell-mediated immune response..$^{98}$

CMs were prepared with Pluronic ${ }^{\circledR}$ F127 as an immunomodulating and stabilizing agent to enhance the stability for controlled drug release and adjuvanticity. ${ }^{94}$ Pluronic, a triblock copolymer of polyethylene oxide and polypropylene oxide (polyethylene oxide-b-polypropylene oxide-b-polyethylene oxide) commonly known as poloxamer, has a variety of pharmaceutical applications and has become one of the most extensively investigated temperature-sensitive materials. ${ }^{99}$ F127 is water soluble and has a good drug release profile, which makes it a potent drug delivery carrier for a variety of therapeutic and bioactive agents. ${ }^{100-104}$ When Westerink et al intranasally immunized antigen-loaded F127/CMs into mice, it significantly increased systemic and mucosal immune responses compared to those of control groups, ${ }^{105}$ suggesting that the stabilization of protein antigens by F127 enhances the immune response of F127/CMs compared to chitosan alone. This study demonstrated a nasal vaccine delivery strategy for enhancement of the immune response via a synergistic 
effect of chitosan and F127. In another study, intraperitoneally and subcutaneously injected F127/cytosine-phosphateguanosine and F127/CM formulations significantly enhanced antigen-specific systemic antibody responses compared to the antigens delivered with cytosine-phosphate-guanosine or CMs alone, ${ }^{106}$ suggesting that F127 might have an adjuvant effect when used in combination with chitosan. Therefore, application of a delivery system that combines adjuvants with various modes of action is beneficial to maximizing immune response.

\section{Limitations of CMs}

Besides the enormous advantages of CMs such as biodegradability, nontoxicity, permeation enhancing effects, and an ability to open the tight junction between epithelial cells as described earlier, there are some limitations as well. Cho and colleagues performed several studies on CMs for vaccine delivery. ${ }^{49,90-94}$ They found that the vaccine-loaded CMs self-aggregated at 2 weeks after preparation, although it was effective in inducing immune responses including cytokine expression in vitro and antigen-specific IgG and IgA responses in vivo after nasal delivery. ${ }^{49,90}$ To make stable and nonaggregated CMs, they used F127 to prepare F127/CMs which showed spherical morphology with no aggregation at an extended period of time after preparation. This was due to the hydrophilic polyethylene oxide chains of F127 that hindered the self-aggregation of CMs. ${ }^{94}$ F127/CMs showed much improved immune activity in vitro and in vivo and also exhibited potential protection against infection compared to CMs alone. ${ }^{94}$

Several other studies described the instability of CMs in acidic media, especially when prepared by the precipitation method. CMs prepared by sodium sulfate precipitation were found to have poor acidic stability. ${ }^{107}$ This acidic instability was initiated by the addition of sodium sulfate to chitosan acetic acid solution which led to an ionic neutralization of the positively charged amine groups of chitosan, providing poorly soluble chitosan derivatives. ${ }^{107}$ After the addition of acid (increasing proton concentration), the equilibrium shifted to the solubilizing range for chitosan, thus dissolving the CMs. ${ }^{30}$ In another study, sulfadiazine-loaded chitosan beads were prepared using tripolyphosphate; however, it was found that the beads had poor mechanical strength. ${ }^{108}$ Collectively, there are some limitations of CMs that can be overcome by modifying the CMs. For example, F127 is a good strategy to improve the stability and mechanical strength of CMs. Additionally, structural modifications of chitosan (eg, thiolated chitosan) might improve the stability and functionality of CMs.

\section{Thiolated CMs as a modified and improved form of a chitosan- based mucosal vaccine carrier}

Thiolated polymers (ie, thiomers) have gained considerable attention - especially for vaccine delivery - because they are one of the most promising polymers with multifunctional properties including strong mucoadhesivity, enhanced permeation effects, protection ability, stability, and enhanced bioavailability of drugs. ${ }^{109-114}$ Among various thiomer-based carriers, thiolated CMs (TCMs) are highly popular because of their strong mucoadhesiveness and ability to control and extend drug release profiles with improved permeation ability. ${ }^{115-119}$ TCMs can be prepared by immobilizing the thiol-bearing chain on the polymeric backbone of chitosan (Figure 3). The strong mucoadhesivity of TCMs is obtained through the formation of disulfide bonds between the thiol groups of TCMs and cysteine-rich subdomains of mucin glycoproteins at the mucosal surface (Figure 4). ${ }^{120}$ The permeability through the mucosal surface can be enhanced by

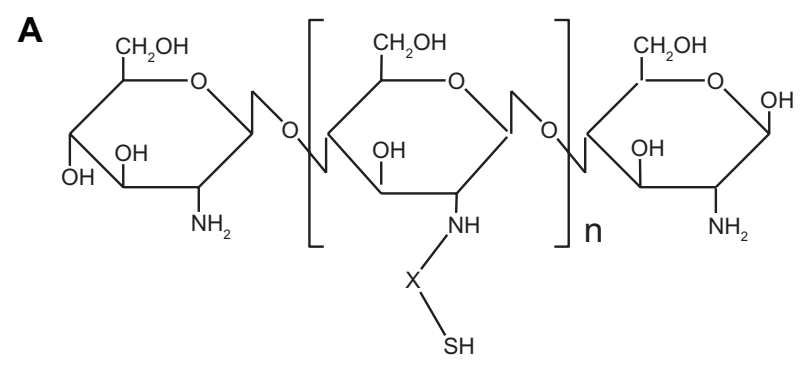

Thiolated chitosan

B

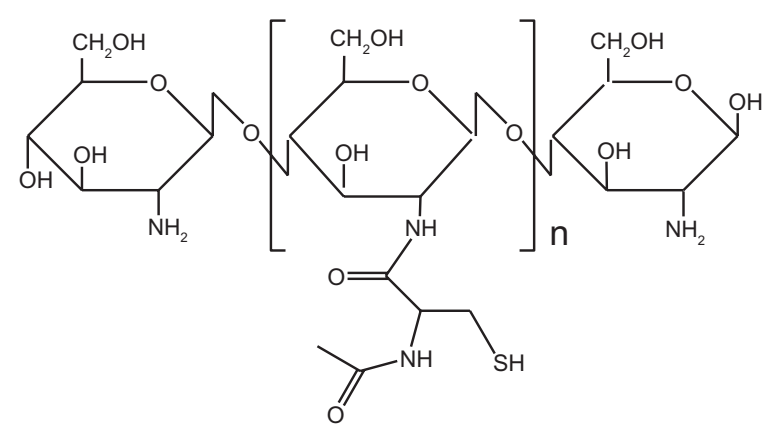

Chitosan-N-acetyl-cysteine

Figure 3 Representative structure of thiolated chitosan: (A) general structure of thiolated chitosan modified by an -SH group (X: linker) and (B) chitosan- $\mathrm{N}$-acetylcysteine (modification of chitosan at the $\mathrm{D}$-glucosamine unit by $\mathrm{N}$-acetyl-cysteine). 

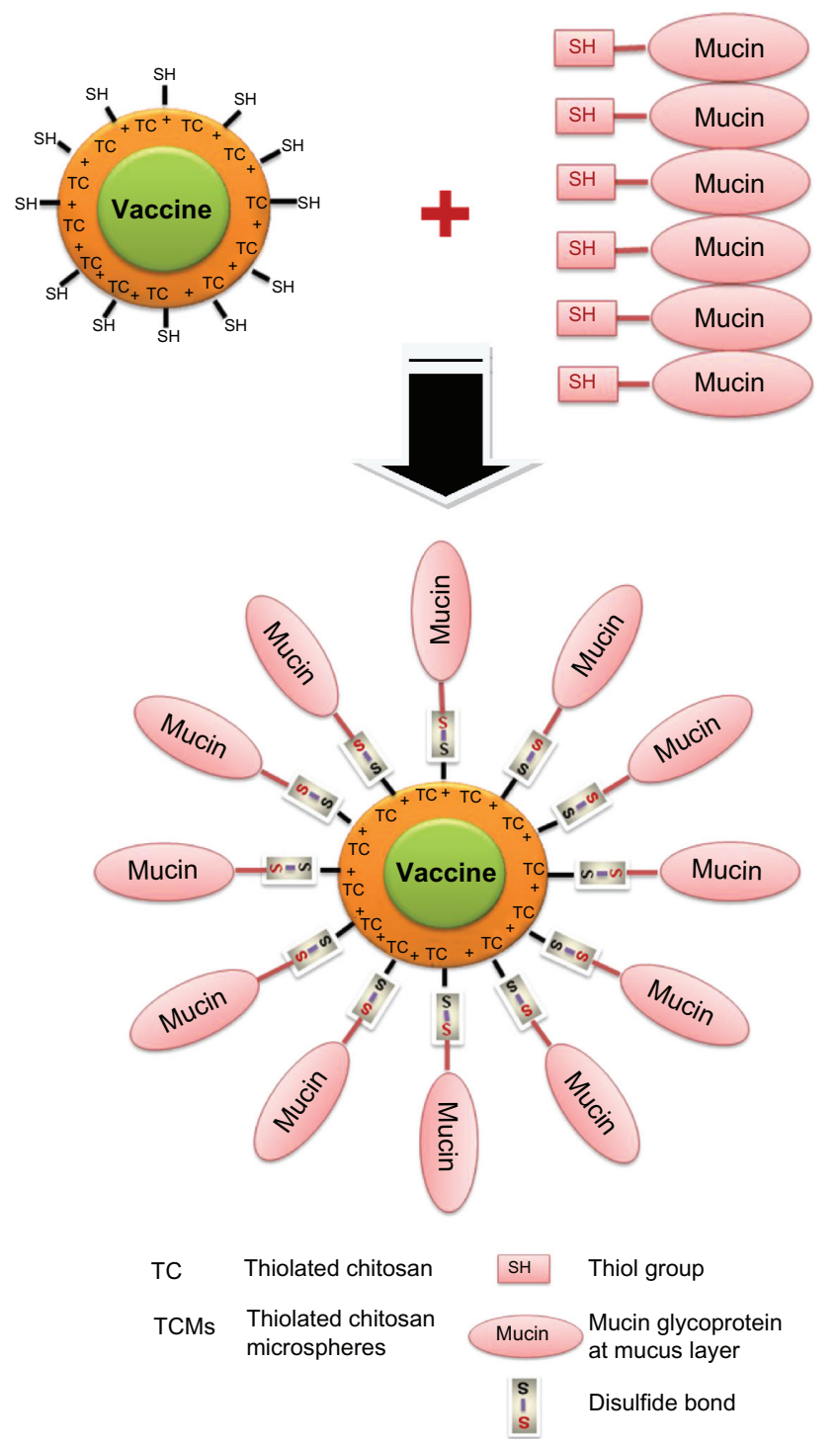

Figure 4 Schematic representation of functional interaction between TCMs and mucin in mucosal vaccine delivery.

Abbreviation: TCM, thiolated chitosan microsphere.

using TCMs instead of unmodified CMs. Increased permeability is achieved by opening the tight junction after the inhibition of protein tyrosine phosphatase, a key enzyme involved in the closing process of tight junction. ${ }^{110}$ Due to the formation of inter- and intramolecular disulfide bonds through TCMs, a compact three-dimensional network is generated which allows controlled drug release and leads to high cohesivity. Moreover, TCMs exhibit a reversible opening of the tight junction, which leads to better permeation effects than unmodified CMs. ${ }^{115,117,120}$ In the case of first-generation thiomers, thiolated chitosan derivatives are prepared by conjugating thiol-bearing aliphatic ligands to the amino groups of chitosan. For example, N-acetyl-cysteine, 6-mecaptonicotinic acid, thioglycolic acid, glutathione, and 2-iminothiolane are the aliphatic thiol-bearing ligands with functional carboxyl groups which form amide bonds with the amino groups of chitosan by carbodiimide to synthesize the thiomers of chitosan. ${ }^{118,121-125} \mathrm{CMs}$ prepared by these thiomers exhibit strong mucoadhesivity, biocompatibility, and enhanced permeability and absorption after oral and nasal administration.

It is important to note that thiomers bearing free thiol groups are relatively unstable in solution because they are prone to oxidize at $\mathrm{pH} \geq 5$, leading to a self-crosslinking of the polymer. Different approaches have been attempted to delay oxidation and inhibit the self-crosslinking reaction. As an example of a next-generation thiomer, the aromatic thiol-bearing ligands are extraordinary candidates for delaying the oxidation process and protecting the thiol groups of the thiolated polymers. ${ }^{126}$ Recently, Bernkop-Schnurch et al performed several studies using aromatic thiol-bearing ligands for the synthesis of S-protected thiolated chitosan and evaluated their efficacy as mucosal drug delivery carriers. ${ }^{109,127,128}$ To prepare the S-protected thiolated chitosan, the thiol-bearing ligand was covalently attached to chitosan as the first step of modification. In the second step, the thiol group of thiolated chitosan was protected by the formation of disulfide bonds with aromatic thiol-bearing ligands. The S-protected thiolated chitosan exhibited improved mucoadhesivity, enhanced permeation effect, inhibited efflux pump, bioavailability, and controlled release profile compared to the corresponding thiolated and unmodified polymers, ${ }^{109,127,128}$ demonstrating that TCMs prepared using S-protected thiolated chitosan are a promising chitosan-based mucoadhesive polymer for the development of various mucosal vaccine delivery systems.

\section{Conclusion and future perspectives}

Among various investigated vaccine carriers, CMs hold enormous promise as a delivery vehicle for both oral and nasal administration. This review has discussed and evaluated various methods for preparation of CMs which could help to design more and better functionalized chitosan-based carrier systems. This study demonstrated that vaccine-loaded CMs could be prepared with suitable and appropriate particle sizes, which is a very important factor in the delivery of the vaccine to the induction site of mucosa-associated lymphoid tissue for proper immune stimulation. Furthermore, both systemic and local immune responses can be induced in a dose- and time-dependent manner through vaccine-loaded CMs. The nontoxic, highly bioavailable, mucoadhesive, and biodegradable nature of chitosan and its particulate 
form is the main reason that it could become a successful vaccine carrier in the near future. Furthermore, the much improved properties of modified CMs (eg, TCMs), such as increased mucoadhesivity, membrane permeability, stability, and controlled/extended release of the encapsulated vaccine, show that they are a promising candidate for a potent vaccine carrier system. Further research and the ability to modify chitosan may improve structural and physicochemical properties, increasing the potential of CM systems. New possibilities in the field of targeted vaccine delivery may be unlocked once various specific ligands (targeting moieties such as mannose and folate) that can be conjugated with chitosan derivatives have been designed and examined for specific interactions with preferred cell types. However, there are many challenges including low physical and mechanical stability, irregular particle size and distribution, and low target specificity that have hindered the efficacy, practical use, and commercialization of CMs. Thus, considering these factors, carefully designed and better functionalized CMs could be prepared for fruitful future application.

\section{Acknowledgments}

This research was supported by the Cooperative Research Program for Agriculture, Science, and Technology Development (PJ 007611) and the Next-Generation BioGreen 21 Program (PJ81272011), Rural Development Administration, Republic of Korea. This work was also partially supported by grants from the Basic Science Research Program through the National Research Foundation of Korea funded by the Ministry of Education, Science, and Technology (2010-0027222, 2010-0003291) and by the Agriculture Research Center Program of the Ministry for Food, Agriculture, Forestry, and Fisheries, Republic of Korea. MA Islam and J Firdous were supported by the Brain Korea 21 Program.

\section{Disclosure}

The authors report no conflicts of interest in this work.

\section{References}

1. Pack DW. Timing is everything. Nat Mater. 2004;3(3):133-134.

2. Giudice EL, Campbell JD. Needle-free vaccine delivery. Adv Drug Deliv Rev. 2006;58(1):68-89.

3. Mitragotri S. Immunization without needles. Nat Rev Immunol. 2005; 5(12):905-916.

4. Quan JS, Jiang HL, Kim EM, et al. pH-sensitive and mucoadhesive thiolated Eudragit-coated chitosan microspheres. Int J Pharm. 2008; 359(1-2):205-210.

5. Mcghee JR, Mestecky J, Dertzbaugh MT, Eldridge JH, Hirasawa M, Kiyono $\mathrm{H}$. The mucosal immune system: from fundamental concepts to vaccine development. Vaccine. 1992;10(2):75-88.
6. Eyles JE, Sharp GJ, Williamson ED, Spiers ID, Alpar HO. Intra nasal administration of poly-lactic acid microsphere co-encapsulated Yersinia pestis subunits confers protection from pneumonic plague in the mouse. Vaccine. 1998;16(7):698-707.

7. Janes KA, Calvo P, Alonso MJ. Polysaccharide colloidal particles as delivery systems for macromolecules. Adv Drug Deliv Rev. 2001;47(1):83-97.

8. Mi FL, Shyu SS, Chen CT, Schoung JY. Porous chitosan microsphere for controlling the antigen release of Newcastle disease vaccine: preparation of antigen-adsorbed microsphere and in vitro release. Biomaterials. 1999;20(17):1603-1612.

9. Artursson P, Lindmark T, Davis SS, Illum L. Effect of chitosan on the permeability of monolayers of intestinal epithelial cells (Caco-2). Pharm Res. 1994;11(9):1358-1361.

10. Illum L, Farraj NF, Davis SS. Chitosan as a novel nasal delivery system for peptide drugs. Pharm Res. 1994;11(8):1186-1189.

11. Sinha VR, Singla AK, Wadhawan S, et al. Chitosan microspheres as a potential carrier for drugs. Int J Pharm. 2004;274(1-2):1-33.

12. Kumar MN, Muzzarelli RA, Muzzarelli C, Sashiwa H, Domb AJ. Chitosan chemistry and pharmaceutical perspectives. Chem Rev. 2004;104(12):6017-6084.

13. Kas HS. Chitosan: properties, preparations and application to microparticulate systems. J Microencapsul. 1997;14(6):689-711.

14. Kato Y, Onishi H, Machida Y. Application of chitin and chitosan derivatives in the pharmaceutical field. Curr Pharm Biotechnol. 2003;4(5):303-309.

15. Singla AK, Chawla M. Chitosan: some pharmaceutical and biological aspects - an update. J Pharm Pharmacol. 2001;53(8): 1047-1067.

16. Gallo JM, Hassan EE. Receptor-mediated magnetic carriers: basis for targeting. Pharm Res. 1988;5(5):300-304.

17. Jameela SR, Kumary TV, Lal AV, Jayakrishnan A. Progesterone-loaded chitosan microspheres: a long acting biodegradable controlled delivery system. J Control Release. 1998;52(1-2):17-24.

18. Jabbal-Gill I, Fisher AN, Rappuoli R, Davis SS, Illum L. Stimulation of mucosal and systemic antibody responses against Bordetella pertussis filamentous haemagglutinin and recombinant pertussis toxin after nasal administration with chitosan in mice. Vaccine. 1998;16(20): 2039-2046.

19. Witschi C, Mrsny RJ. In vitro evaluation of microparticles and polymer gels for use as nasal platforms for protein delivery. Pharm Res. 1999;16(3):382-390.

20. Alameh M, De Jesus D, Jean M, et al. Low molecular weight chitosan nanoparticulate system at low N:P ratio for nontoxic polynucleotide delivery. Int J Nanomedicine. 2012;7:1399-1414.

21. Jean M, Alameh M, De Jesus D, et al. Chitosan-based therapeutic nanoparticles for combination gene therapy and gene silencing of in vitro cell lines relevant to type 2 diabetes. Eur J Pharm Sci. 2012;45(1-2):138-149.

22. Luo Y, Zhai X, Ma C, et al. An inhalable $\beta 2$-adrenoceptor liganddirected guanidinylated chitosan carrier for targeted delivery of siRNA to lung. J Control Release. 2012;162(1):28-36.

23. Yang Y, Liu X, Zhang D, et al. Chitosan/VEGF-sIRNA nanoparticle for gene silencing. J Control Release. 2011;152(Suppl 1):e160-e161.

24. Dash M, Chiellini F, Ottenbrite RM, Chiellini E. Chitosan - a versatile semi-synthetic polymer in biomedical applications. Prog Polym Sci. 2011;36(8):981-1014.

25. Xu J, McCarthy SP, Gross RA, Kaplan DL. Chitosan film acylation and effects on biodegradability. Macromolecules. 1996;29(10): 3436-3440.

26. Yang YM, Hu W, Wang XD, Gu XS. The controlling biodegradation of chitosan fibers by $\mathrm{N}$-acetylation in vitro and in vivo. J Mater Sci Mater Med. 2007;18(11):2117-2121.

27. Kean T, Thanou M. Biodegradation, biodistribution and toxicity of chitosan. Adv Drug Deliv Rev. 2010;62(1):3-11.

28. Arai K, Kineemaki T, Fujita T. Toxicity of chitosan. Bull Tokai Reg Fish Res Lab. 1968;56:89-94. 
29. Hirano S, Seino H, Akiyama Y, Nonaka I. Biocompatibility of chitosan by oral and intravenous administrations. Polym Mater Sci Eng. 1988;59:897-901.

30. Illum L. Chitosan and its use as a pharmaceutical excipient. Pharm Res. 1998;15(9):1326-1331.

31. Wedmore I, McManus JG, Pusateri AE, Holcomb J. A special report on the chitosan-based hemostatic dressing: experience in current combat operations. J Trauma. 2006;60(3):655-658.

32. Shaji J, Jain V, Lodha S. Chitosan: a novel pharmaceutical excipient. Int J Pharm Appl Sci. 2010;1(1):11-28.

33. Jumaa M, Furkert FH, Muller BW. A new lipid emulsion formulation with high antimicrobial efficacy using chitosan. Eur J Pharm Biopharm. 2002;53(1):115-123.

34. Guo Z, Chen R, Xing R, et al. Novel derivatives of chitosan and their antifungal activities in vitro. Carbohydr Res. 2006;341(3):351-354.

35. Pujals G, Sune-Negre JM, Perez P, et al. In vitro evaluation of the effectiveness and cytotoxicity of meglumine antimoniate microspheres produced by spray drying against Leishmania infantum. Parasitol Res. 2008;102(6):1243-1247.

36. Rao SB, Sharma CP. Use of chitosan as a biomaterial: studies on its safety and hemostatic potential. J Biomed Mater Res. 1997;34(1):21-28.

37. Gades MD, Stern JS. Chitosan supplementation and fecal fat excretion in men. Obes Res. 2003;11(5):683-688.

38. Sonaje K, Lin YH, Juang JH, Wey SP, Chen CT, Sung HW. In vivo evaluation of safety and efficacy of self-assembled nanoparticles for oral insulin delivery. Biomaterials. 2009;30(12):2329-2339.

39. Wang LY, Gu YH, Su ZG, Ma GH. Preparation and improvement of release behavior of chitosan microspheres containing insulin. Int $J$ Pharm. 2006;311(1-2):187-195.

40. Thanou M, Verhoef JC, Junginger HE. Oral drug absorption enhancement by chitosan and its derivatives. Adv Drug Deliv Rev. 2001;52(2):117-126.

41. Thanou M, Verhoef JC, Junginger HE. Chitosan and its derivatives as intestinal absorption enhancers. Adv Drug Deliv Rev. 2001;50(Suppl 1): S91-S101.

42. Borchard G, Luessen HL, De Boer AG, Verhoef JC, Lehr CM, Junginger HE. The potential of mucoadhesive polymers in enhancing intestinal peptidedrug absorption. III: effects of chitosanglutamate and carbomer on epithelial tight junctions in vitro. J Control Release. 1996;39:131-138.

43. Patil S, Babbar A, Mathur R, Mishra A, Sawant K. Mucoadhesive chitosan microspheres of carvedilol for nasal administration. J Drug Target. 2010;18(4):321-331.

44. Joscelyne SM, Tragardh G. Membrane emulsification - a literature review. J Memb Sci. 2000;169(1):107-117.

45. Yoo JW, Irvine DJ, Discher DE, Mitragotri S. Bio-inspired, bioengineered and biomimetic drug delivery carriers. Nat Rev Drug Discov. 2011;10(7):521-535.

46. Jayakumar R, Chennazhi KP, Muzzarelli RA, Tamura H, Nair SV, Selvamurugan N. Chitosan conjugated DNA nanoparticles in gene therapy. Carbohydr Polym. 2010;79(1):1-8.

47. Jreyssaty C, Shi Q, Wang H, et al. Efficient nonviral gene therapy using folate-targeted chitosan-DNA nanoparticles in vitro. ISRN Pharm. 2012;2012:369270.

48. Tong H, Shi Q, Fernandes JC, Li L, Dai K, Zhang X. Progress and prospects of chitosan and its derivatives as non-viral gene vectors in gene therapy. Curr Gene Ther. 2009;9(6):495-502.

49. Jiang HL, Park IK, Shin NR, et al. In vitro study of the immune stimulating activity of an atrophic [correction of athrophic] rhinitis vaccine associated to chitosan microspheres. Eur J Pharm Biopharm. 2004;58(3):471-476.

50. Lim LY, Wan LSC, Thai PY. Chitosan microspheres prepared by emulsification and ionotropic gelation. Drug Dev Ind Pharm. 1997;23(10):981-985.

51. Shu XZ, Zhu KJ. Chitosan/gelatin microspheres prepared by modified emulsification and ionotropic gelation. J Microencapsul. 2001;18(2):237-245.
52. Daly MM, Knorr D. Chitosan-alginate complex coacervate capsules: effects of calcium chloride, plasticizers, and polyelectrolytes on mechanical stability. Biotechnol Prog. 1988;4(2):76-81.

53. Nishioka Y, Kyotani S, Okamura M, et al. Release characteristics of cisplatin chitosan microspheres and effect of containing chitin. Chem Pharm Bull (Tokyo). 1990;38(10):2871-2873.

54. Ohya Y, Takei T, Kobayashi H, Ouchi T. Release behaviour of 5-fluorouracil from chitosan-gel microspheres immobilizing 5-fluorouracil derivative coated with polysaccharides and their cell specific recognition. J Microencapsul. 1993;10(1):1-9.

55. Pavanetto F, Perugini P, Conti B, Modena T, Genta I. Evaluation of process parameters involved in chitosan microsphere preparation by the o/w/o multiple emulsion method. J Microencapsul. 1996;13(6):679-688.

56. Thanoo BC, Sunny MC, Jayakrishnan A. Cross-linked chitosan microspheres: preparation and evaluation as a matrix for the controlled release of pharmaceuticals. J Pharm Pharmacol. 1992;44(4):283-286.

57. Orienti I, Aiedeh K, Gianasi E, Ponti C, Zecchi V. Chitosanindomethacin conjugates. Effect of different substituents on the polysaccharide molecule on drug release. Arch Pharm Pharm Med Chem. 1996;329(5):245-250.

58. Mi FL, Tan YC, Liang HF, Sung HW. In vivo biocompatibility and degradability of a novel injectable-chitosan-based implant. Biomaterials. 2002;23(1):181-191.

59. van der Lubben IM, van Opdorp FA, Hengeveld MR, et al. Transport of chitosan microparticles for mucosal vaccine delivery in a human intestinal M-cell model. J Drug Target. 2002;10(6):449-456.

60. Yoo JS, Kim YJ, Kim SH, Choi SH. Study on genipin: a new alternative natural crosslinking agent for fixing heterogaft tissue. Korean J Thorac Cardiovasc Surg. 2011;44(3):197-207.

61. Tokumitsu H, Ichikawa H, Fukumori Y. Chitosan-gadopentetic acid complex nanoparticles for gadolinium neutron-capture therapy of cancer: preparation by novel emulsion-droplet coalescence technique and characterization. Pharm Res. 1999;16(12):1830-1835.

62. Mitra A, Dey B. Chitosan microspheres in novel drug delivery systems. Indian J Pharm Sci. 2011;73(4):355-366.

63. Ozbas-Turan S, Akbuga J, Aral C. Controlled release of interleukin-2 from chitosan microspheres. J Pharm Sci. 2002;91(5):1245-1251.

64. Mitra S, Gaur U, Ghosh PC, Maitra AN. Tumor targeted delivery of encapsulated dextran-doxorubicin conjugate using chitosan nanoparticles as carrier. J Control Release. 2001;74(1-3):317-323.

65. Agnihotri SA, Aminabhavi TM. Controlled release of clozapine through chitosan microparticles prepared by a novel method. J Control Release. 2004;96(2):245-259.

66. Bogataj M, Mrhar A, Grabnar I, et al. The influence of magnesium stearate on the characteristics of mucoadhesive microspheres. J Microencapsul. 2000;17(4):499-508.

67. He P, Davis SS, Illum L. Sustained release chitosan microspheres prepared by novel spray drying methods. J Microencapsul. 1999;16(3):343-355.

68. van der Lubben IM, Verhoef JC, van Aelst AC, Borchard G, Junginger HE. Chitosan microparticles for oral vaccination: preparation, characterization and preliminary in vivo uptake studies in murine Peyer's patches. Biomaterials. 2001;22(7):687-694.

69. Ahire VJ, Sawant KK, Doshi JB, Ravetkar SD. Chitosan microparticles as oral delivery system for tetanus toxoid. Drug Dev Ind Pharm. 2007;33(10):1112-1124.

70. van der Lubben IM, Kersten G, Fretz MM, Beuvery C, Verhoef JC, Junginger HE. Chitosan microparticles for mucosal vaccination against diphtheria: oral and nasal efficacy studies in mice. Vaccine. 2003; 21(13-14):1400-1408.

71. van der Lubben IM, Konings FA, Borchard G, Verhoef JC, Junginger HE. In vivo uptake of chitosan microparticles by murine Peyer's patches: visualization studies using confocal laser scanning microscopy and immunohistochemistry. J Drug Target. 2001;9(1):39-47.

72. Hori M, Onishi H, Machida Y. Evaluation of Eudragit-coated chitosan microparticles as an oral immune delivery system. Int J Pharm. $2005 ; 297(1-2): 223-234$. 
73. Premaletha K, Licy CD, Jose S, Saraladevi A, Shirwaikar A, Shirwaikar A. Formulation, characterization and optimization of hepatitis B surface antigen (HBsAg)-loaded chitosan microspheres for oral delivery. Pharm Dev Technol. 2012;17(2):251-258.

74. Uddin AN, Bejugam NK, Gayakwad SG, Akther P, D'Souza MJ. Oral delivery of gastro-resistant microencapsulated typhoid vaccine. J Drug Target. 2009;17(7):553-560.

75. Chiou CJ, Tseng LP, Deng MC, et al. Mucoadhesive liposomes for intranasal immunization with an avian influenza virus vaccine in chickens. Biomaterials. 2009;30(29):5862-5868.

76. Pine $\mathrm{S}$, Barackman J, Ott G, O’Hagan D. Intranasal immunization with influenza vaccine and a detoxified mutant of heat labile enterotoxin from Escherichia coli (LTK63). J Control Release. 2002;85(1-3): 263-270.

77. Carr RM, Lolachi CM, Albaran RG, Ridley DM, Montgomery PC, O'Sullivan NL. Nasal-associated lymphoid tissue is an inductive site for rat tear IgA antibody responses. Immunol Invest. 1996;25(5-6): 387-396.

78. Cleary PP, Zhang Y, Park HS. Nasal associated lymphoid tissue and $\mathrm{M}$ cells, a window to persistent streptococcal infections. Indian J Med Res. 2004;119 Suppl:57-60.

79. Debertin AS, Tschernig T, Tonjes H, Kleemann WJ, Troger HD, Pabst R. Nasal-associated lymphoid tissue (NALT): frequency and localization in young children. Clin Exp Immunol. 2003;134(3):503-507.

80. Gill RF, Pirockinaite G, O’Sullivan NL, Montgomery PC. Nasalassociated lymphoid tissue is not an absolute requirement for the induction of rat tear IgA antibody responses. Curr Eye Res. 2010;35(1):1-8.

81. Hopkins S, Fisher G, Kraehenbuhl JP, Velin D. Nasal-associated lymphoid tissue - a site for vaccination and pathogen entry. STP Pharm Sci. 1998;8(1):47-51.

82. Liang B, Hyland L, Hou S. Nasal-associated lymphoid tissue is a site of long-term virus-specific antibody production following respiratory virus infection of mice. J Virol. 2001;75(11):5416-5420.

83. Owen SJ, Batzloff M, Chehrehasa F, et al. Nasal-associated lymphoid tissue and olfactory epithelium as portals of entry for Burkholderia pseudomallei in murine melioidosis. J Infect Dis. 2009;199(12): 1761-1770.

84. Zuercher AW, Coffin SE, Thurnheer MC, Fundova P, Cebra JJ. Nasal-associated lymphoid tissue is a mucosal inductive site for virus-specific humoral and cellular immune responses. J Immunol. 2002;168(4):1796-1803.

85. McNeela EA, O'Connor D, Jabbal-Gill I, et al. A mucosal vaccine against diphtheria: formulation of cross reacting material (CRM197) of diphtheria toxin with chitosan enhances local and systemic antibody and Th2 responses following nasal delivery. Vaccine. 2000;19(9-10):1188-1198.

86. Donovan MD, Flynn GL, Amidon GL. Absorption of polyethylene glycols 600 through 2000: the molecular weight dependence of gastrointestinal and nasal absorption. Pharm Res. 1990;7(8):863-868.

87. Sarkar MA. Drug metabolism in the nasal mucosa. Pharm Res. 1992;9(1):1-9.

88. Baudner BC, Giuliani MM, Verhoef JC, Rappuoli R, Junginger HE, Giudice GE. The concomitant use of the LTK63 mucosal adjuvant and of chitosan-based delivery system enhances the immunogenicity and efficacy of intranasally administered vaccines. Vaccine. 2003;21(25-26):3837-3844.

89. Illum L, Jabbal-Gill I, Hinchcliffe M, Fisher AN, Davis SS. Chitosan as a novel nasal delivery system for vaccines. Adv Drug Deliv Rev. 2001;51(1-3):81-96.

90. Kang ML, Kang SG, Jiang HL, et al. In vivo induction of mucosal immune responses by intranasal administration of chitosan microspheres containing Bordetella bronchiseptica DNT. Eur J Pharm Biopharm. 2006;63(2):215-220.

91. Jiang HL, Kang ML, Quan JS, et al. The potential of mannosylated chitosan microspheres to target macrophage mannose receptors in an adjuvant-delivery system for intranasal immunization. Biomaterials. 2008;29(12):1931-1939.
92. Jiang HL, Park IK, Kang ML, et al. Immune stimulating activity of an atrophic rhinitis vaccine associated to pegylated chitosan microspheres in vitro. Polym Adv Technol. 2007;18(3):220-225.

93. Jiang HL, Park IK, Shin NR, Yoo HS, Akaike T, Cho CS. Controlled release of Bordetella bronchiseptica dermonecrotoxin (BBD) vaccine from BBD-loaded chitosan microspheres in vitro. Arch Pharm Res. 2004;27(3):346-350.

94. Kang ML, Jiang HL, Kang SG, et al. Pluronic F127 enhances the effect as an adjuvant of chitosan microspheres in the intranasal delivery of Bordetella bronchiseptica antigens containing dermonecrotoxin. Vaccine. 2007;25(23):4602-4610.

95. Soane RJ, Frier M, Perkins AC, Jones NS, Davis SS, Illum L. Evaluation of the clearance characteristics of bioadhesive systems in humans. Int J Pharm. 1999;178(1):55-65.

96. Soane RJ, Hinchcliffe M, Davis SS, Illum L. Clearance characteristics of chitosan based formulations in the sheep nasal cavity. Int J Pharm. 2001;217(1-2):183-191.

97. Baudner BC, Verhoef JC, Giuliani MM, et al. Protective immune responses to meningococcal $\mathrm{C}$ conjugate vaccine after intranasal immunization of mice with the LTK63 mutant plus chitosan or trimethyl chitosan chloride as novel delivery platform. $J$ Drug Target. 2005;13(8-9):489-498.

98. Kumar M, Behera AK, Lockey RF, et al. Intranasal gene transfer by chitosan-DNA nanospheres protects BALB/c mice against acute respiratory syncytial virus infection. Hum Gene Ther. 2002;13(12): $1415-1425$.

99. Wang PL, Johnston TP. Enhanced stability of two model proteins in an agitated solution environment using poloxamer 407.J Parenter Sci Technol. 1993;47(4):183-189.

100. Morikawa K, Okada F, Hosokawa M, Kobayashi K. Enhancement of therapeutic effects of recombinant interleukin-2 on a transplantable rat fibrosarcoma by the use of a sustained release vehicle, pluronic gel. Cancer Res. 1987;47(1):37-41.

101. Kang ML, Cho CS, Yoo HS. Application of chitosan microspheres for nasal delivery of vaccines. Biotechnol Adv. 2009;27(6):857-865.

102. Miyazaki S, Tobiyama T, Takada M, Attwood D. Percutaneous absorption of indomethacin from pluronic F127 gels in rats. J Pharm Pharmacol. 1995;47(6):455-457.

103. Veyries ML, Couarraze G, Geiger S, et al. Controlled release of vancomycin from poloxamer 407 gels. Int J Pharm. 1999;192(2):183-193.

104. Zhang L, Parsons DL, Navarre C, Kompella UB. Development and in-vitro evaluation of sustained release poloxamer 407 (P407) gel formulations of ceftiofur. $J$ Control Release. 2002;85(1-3):73-81.

105. Westerink MA, Smithson SL, Srivastava N, Blonder J, Coeshott C, Rosenthal GJ. ProJuvant (Pluronic F127/chitosan) enhances the immune response to intranasally administered tetanus toxoid. Vaccine. 2001;20(5-6):711-723.

106. Coeshott CM, Smithson SL, Verderber E, et al. Pluronic F127based systemic vaccine delivery systems. Vaccine. 2004;22(19): 2396-2405.

107. Berthold A, Cremer K, Kreuter J. Preparation and characterization of chitosan microspheres as drug carrier for prednisolone sodium phosphate as model for antiinflammatory drugs. $J$ Control Release. 1996;39(1):17-25.

108. Bodmeier R, Oh KH, Pramar Y. Preparation and evaluation of drug-containing chitosan beads. Drug Dev Ind Pharm. 1989;15(9): 1475-1494.

109. Dunnhaupt S, Barthelmes J, Thurner CC, Waldner C, Sakloetsakun D, Bernkop-Schnurch A. S-protected thiolated chitosan: synthesis and in vitro characterization. Carbohydr Polym. 2012;90(2):765-772.

110. Hauptstein S, Bernkop-Schnurch A. Thiomers and thiomer-based nanoparticles in protein and DNA drug delivery. Expert Opin Drug Deliv. 2012;9(9):1069-1081.

111. Islam MA, Bajracharya P, Kang SK, et al. Mucoadhesive alginate/ poly (L-lysine)/thiolated alginate microcapsules for oral delivery of Lactobacillus salivarius 29. J Nanosci Nanotechnol. 2011;11(8): 7091-7095. 
112. Islam MA, Jiang HL, Quan JS, et al. Mucoadhesive and $\mathrm{pH}$-sensitive thiolated Eudragit microspheres for oral delivery of Pasteurella multocida antigens containing dermonecrotoxin. J Nanosci Nanotechnol. 2011;11(5):4174-4181.

113. Lee WJ, Cha S, Shin M, Islam MA, Cho CS, Yoo HS. Induction of Th1 polarized immune responses by thiolated Eudragit-coated F4 and F18 fimbriae of enterotoxigenic Escherichia coli. Eur J Pharm Biopharm. 2011;79(2):226-231.

114. Lee WJ, Cha S, Shin M, et al. Efficacy of thiolated Eudragit microspheres as an oral vaccine delivery system to induce mucosal immunity against enterotoxigenic Escherichia coli in mice. Eur J Pharm Biopharm. 2012;81(1):43-48.

115. Iqbal J, Shahnaz G, Perera G, Hintzen F, Sarti F, Bernkop-Schnurch A. Thiolated chitosan: development and in vivo evaluation of an oral delivery system for leuprolide. Eur J Pharm Biopharm. 2012;80(1): 95-102.

116. Millotti G, Samberger C, Frohlich E, Sakloetsakun D, BernkopSchnurch A. Chitosan-4-mercaptobenzoic acid: synthesis and characterization of a novel thiolated chitosan. J Mater Chem. 2010;20(12):2432-2440.

117. Shahnaz G, Vetter A, Barthelmes J, et al. Thiolated chitosan nanoparticles for the nasal administration of leuprolide: bioavailability and pharmacokinetic characterization. Int J Pharm. 2012;428(1-2): 164-170.

118. Talaei F, Azizi E, Dinarvand R, Atyabi F. Thiolated chitosan nanoparticles as a delivery system for antisense therapy: evaluation against EGFR in T47D breast cancer cells. Int $J$ Nanomedicine. 2011;6:1963-1975.

119. Werle M, Bernkop-Schnurch A. Thiolated chitosans: useful excipients for oral drug delivery. J Pharm Pharmacol. 2008;60(3):273-281.
120. Bernkop-Schnurch A, Kast CE, Guggi D. Permeation enhancing polymers in oral delivery of hydrophilic macromolecules: thiomer/ GSH systems. J Control Release. 2003;93(2):95-103.

121. Millotti G, Perera G, Vigl C, Pickl K, Sinner FM, Bernkop-Schnurch A. The use of chitosan-6-mercaptonicotinic acid nanoparticles for oral peptide drug delivery. Drug Deliv. 2011;18(3):190-197.

122. Millotti G, Samberger C, Frohlich E, Bernkop-Schnurch A. Chitosangraft-6-mercaptonicotinic acid: synthesis, characterization, and biocompatibility. Biomacromolecules. 2009;10(11):3023-3027.

123. Saboktakin MR, Tabatabaie RM, Maharramov A, Ramazanov MA. Development and in vitro evaluation of thiolated chitosanpoly(methacrylic acid) nanoparticles as a local mucoadhesive delivery system. Int J Biol Macromol. 2011;48(3):403-407.

124. Saremi S, Atyabi F, Akhlaghi SP, Ostad SN, Dinarvand R. Thiolated chitosan nanoparticles for enhancing oral absorption of docetaxel: preparation, in vitro and ex vivo evaluation. Int $J$ Nanomedicine. 2011;6:119-128.

125. Dunnhaupt S, Barthelmes J, Hombach J, Sakloetsakun D, Arkhipova V, Bernkop-Schnurch A. Distribution of thiolated mucoadhesive nanoparticles on intestinal mucosa. Int J Pharm. 2011;408(1-2):191-199.

126. Burner U, Jantschko W, Obinger C. Kinetics of oxidation of aliphatic and aromatic thiols by myeloperoxidase compounds I and II. FEBS Lett. 1999;443(3):290-296.

127. Dunnhaupt S, Barthelmes J, Iqbal J, et al. In vivo evaluation of an oral drug delivery system for peptides based on S-protected thiolated chitosan. J Control Release. 2012;160(3):477-485.

128. Dunnhaupt S, Barthelmes J, Rahmat D, et al. S-protected thiolated chitosan for oral delivery of hydrophilic macromolecules: evaluation of permeation enhancing and efflux pump inhibitory properties. $\mathrm{Mol}$ Pharm. 2012;9(5):1331-1341.
International Journal of Nanomedicine

\section{Publish your work in this journal}

The International Journal of Nanomedicine is an international, peerreviewed journal focusing on the application of nanotechnology in diagnostics, therapeutics, and drug delivery systems throughout the biomedical field. This journal is indexed on PubMed Central, MedLine, CAS, SciSearch $®$, Current Contents ${ } /$ Clinical Medicine,

\section{Dovepress}

Journal Citation Reports/Science Edition, EMBase, Scopus and the Elsevier Bibliographic databases. The manuscript management system is completely online and includes a very quick and fair peer-review system, which is all easy to use. Visit http://www.dovepress.com/ testimonials.php to read real quotes from published authors. 